\title{
Antimicrobial stewardship programmes: the need for wider engagement
}

\author{
Esmita Charani, Alison H Holmes
}

Department of Infectious Diseases and Immunity, Imperial College London, The Centre for Infection Prevention and Management, London, UK

\section{Correspondence to} Esmita Charani, Department of Infectious Diseases and Immunity, Imperial College London, The Centre for Infection Prevention and Management; 8th Floor, The Commonwealth Building, Du Cane Rd, London W12 ONN, UK e.charani@imperial.ac.uk

Received 30 August 2013 Accepted 30 August 2013 Published Online First

17 September 2013

\section{SLinked}

- http://dx.doi.org/10.1136/ bmjqs-2012-001791

To cite: Charani $\mathrm{E}_{\text {, }}$ Holmes AH. BMJ Qual Saf 2013:22:885-887.
Antimicrobial resistance has been recognised as a major global health threat ${ }^{1}$ and is now on the political agenda with world leaders recognising the necessity to act to preserve the potency of antimicrobial agents and invest funds to discover new ones. $^{2}$ Despite the majority of antimicrobial prescribing and consumption occurring in primary care settings, ${ }^{3}$ hospitalised populations experience the full force of antimicrobial resistance and difficult-to-treat multidrug resistant organisms. ${ }^{4}$

To optimise antimicrobial prescribing, reduce healthcare associated infections and minimise the emergence of antimicrobial resistance, hospitals in both developed and developing healthcare systems are increasingly implementing initiatives ranging from targeted interventions $^{5}$ to antimicrobial stewardship programmes. ${ }^{67}$ Antimicrobial stewardship is the umbrella term used to define comprehensive quality improvement activities that together represent a cohesive programme aiming to optimise the use of antimicrobials, improve patient outcomes, reduce the spread and development of antimicrobial resistance and reduce the incidence of healthcare acquired infections.

To assist antimicrobial stewardship initiatives, governments, policy makers and researchers have attempted to draw up recommendations for optimal practice. ${ }^{8-10}$ The prevailing expectation of healthcare systems is to deliver high quality care in the face of increasing financial cuts and increasing numbers of patients treated. Therefore, a pragmatic approach to developing and implementing sustainable interventions in antimicrobial stewardship is required. The challenge of implementing antimicrobial stewardship programmes is not limited to large teaching hospitals and academic centres in the developed world and it follows that the solutions to these problems cannot be limited to them. The increasing globalisation of the world and population mobility ensures the rapid spread of new resistant organisms and infectious diseases ${ }^{11}$ making them shared global problems. It is therefore encouraging to see antimicrobial stewardship initiatives being implemented across the globe. $^{71213}$

\section{EMBEDDING PRACTICE WITHIN EXISTING SYSTEMS AND RESOURCES}

Wide disparities exist in the availability of resources to implement antimicrobial stewardship initiatives in hospitals in both developed and developing healthcare systems. As an example of this disparity, Andersen and Knudsen report in the current issue of this journal ${ }^{12}$ an intervention they implemented in a 500-bed Danish University hospital that did not have onsite clinical microbiology service or staff. They report on the steps undertaken to tackle multidrug resistant infection. In the absence of an on-site antimicrobial stewardship programme, they report on their successful collaboration with a programme at a larger hospital. In a different, recently published study, from the other side of the world in Vietnam, Wertheim and colleagues report that limited resources prevent the enforcement of existing policies on antimicrobial resistance. ${ }^{13}$ Faced with a different challenge to the Danish hospital, the team in Vietnam also looked for local solutions to their problem. These papers highlight the challenges that healthcare organisations face when implementing antimicrobial stewardship programmes across the globe. It is unlikely that there will ever be enough infectious disease specialists in any healthcare system (ie, developing or developed) to drive all the 
antimicrobial stewardship programmes. Faced with the problem of insufficient on-site resources to implement stewardship programmes as typically seen in large teaching hospitals, other options may be used. The companion paper to this editorial reports addressing this gap in resource by:

1. Partnering with a larger hospital with an established stewardship programme

2. Developing targeted quality improvement intervention for a common and recurring problem related to the overuse of antimicrobials.

These options are not mutually exclusive nor are they the only options available. They represent a positive effort in trying to bring about quality improvement in antimicrobial stewardship. Allocating existing resources effectively will require identifying and engaging with key stakeholders, identifying committed influential clinical leads and embedding standards of practice for antimicrobial stewardship programmes within existing pathways.

The cornerstone of all antimicrobial stewardship programmes consists of monitoring practice, establishing a team and developing and implementing interventions focused on antimicrobial practice ${ }^{10}$ and using organisational infrastructure. The key to the success of these programmes lies in bringing together the right team of healthcare professionals and managers to support and monitor best practice. But these teams can be local and embedded within existing specialties. Given the increased regulation and public interest in healthcare acquired infections, antimicrobial stewardship programmes should be a high priority for all healthcare organisations. Optimising the use of antimicrobials is an essential aspect of patient safety. A critical step in prioritising antimicrobial stewardship is to embed it within existing local patient safety programmes. To successfully achieve this requires leadership and teamwork ${ }^{14}$ to drive the programmes and ensure they are implemented locally within specialties and existing cultural structures of hospitals.

\section{Wider engagement of clinical staff}

Antimicrobial stewardship is multidisciplinary and invariably involves very different disciplines depending on the healthcare systems, cultures and continents where it is implemented..$^{5} 9$ A misconception is perhaps that stewardship activities have to be driven by core medical microbiology and infectious diseases specialties. However, as illustrated by Anderson and colleagues, where this expertise is not consistently provided, other healthcare professionals and specialties can initiate activities and develop programmes with contribution from specialists that effectively bring about a positive change in antimicrobial prescribing and infection management programmes. Perhaps considering antimicrobial stewardship as a patient safety and availability of care issue, it should not be restricted to specialists. Recognising the potential contribution of existing staff can help address the challenges in implementing the antimicrobial stewardship programmes in hospitals.

The key frontline healthcare professionals who have a role in antimicrobial stewardship are physicians, nurses and pharmacists. Yet, across Europe and many parts of the world, nurses and pharmacists are not recognised as being important and involved in the stewardship activities and it is rare to find intervention studies in this field where all three groups have representation. This omission is more marked in the case of nurses who make up the bulk of the healthcare work force and are directly responsible in the administration and monitoring of antimicrobial therapy in healthcare settings across the world. When colleagues report on being 'resource poor' in terms of enforcing stewardship activities, it is important to consider whether they have taken into account the potential contribution that the nursing and pharmacy healthcare professionals can make to the antimicrobial stewardship activities.

The technical expertise that specialist infectious disease physicians, pharmacists and medical microbiology teams bring to interventions in antimicrobial stewardship is of critical importance, but these can be supplemented and supported by the wider workforce who are directly involved in the treatment of patients with infections. All healthcare professionals can be involved in having an active role in recognising, implementing and sustaining the principal aims of antimicrobial stewardship programmes. This is an important point to consider since unlike other areas of disease management, the treatment of infection occurs within all specialties, and physicians, nurses and pharmacists of all specialties will have patients under their care who will require antimicrobial therapy. Research has shown that involving and being mindful of local cultures, for example, within specialties, is an important facet of successful interventions. ${ }^{13} 15$ This involves active inclusion and participation of local leaders in the stewardship teams to ensure that stewardship programmes are sustainably embedded into local practice. Wider engagement with clinical specialties is paramount in order to develop sustainable and effective interventions and to embed the key components of stewardship into the local patient safety programmes.

Contributors Both authors equally contributed to the submission of this paper.

Competing interests None.

Provenance and peer review Not commissioned; internally peer reviewed.

\section{REFERENCES}

1 Leung E, Weil DE, Raviglione M, et al. The WHO policy package to combat antimicrobial resistance. Bull World Health Organ 2001;89:390-2. 
2 G8 Ministers pledge to act on antibiotic resistance. Financial Times 2013. http://www.ft.com/cms/s/0/172341bc-d428-11e2a464-00144feab7de.html\#axzz2WqdADmif

3 van Bijnen EM, den Heijer CD, Paget WJ, et al. The appropriateness of prescribing antibiotics in the community in Europe: study design. BMC Infect Dis 2011;11:293.

4 Wiegand PN, Nathwani D, Wilcox MH, et al. Clinical and economic burden of Clostridium difficile infection in Europe: a systematic review of healthcare-facility-acquired infection. J Hosp Infect 2012;81:1-14.

5 Davey P, Brown E, Charani E, et al. Interventions to improve antibiotic prescribing practices for hospital inpatients. Cochrane Database Syst Rev 2013;(4):CD003543.

6 Tamma PD, Cosgrove SE. Antimicrobial stewardship. Infect Dis Clin North Am 2011;25:245-60.

7 Ghafur A, Mathai D, Muruganathan A, et al. The Chennai declaration: a roadmap to tackle The Challenge of antimicrobial resistance. Indian J Cancer 2013;50:71-3.

8 Ashiru-Oredope D, Sharland M, Charani E, et al. J Improving the quality of antibiotic prescribing in the NHS by developing a new Antimicrobial Stewardship Program: Start Smart-Then Focus. ARHAI Antimicrobial Stewardship Group. J Antimicrob Chemother 2012;67(Suppl 1):i51-63.

9 Cooke J, Alexander K, Charani E, et al. Antimicrobial stewardship: an evidence-based, antimicrobial self-assessment toolkit (ASAT) for acute hospitals. J Antimicrob Chemother 2010;65:2669-73

10 Society for Healthcare Epidemiology of America; Infectious Diseases Society of America; Pediatric Infectious Diseases Society. Policy statement on antimicrobial stewardship by the Society for Healthcare Epidemiology of America (SHEA), the Infectious Diseases Society of America (IDSA), and the Pediatric Infectious Diseases Society (PIDS). Infect Control Hosp Epidemiol 2012;33:322-7.

11 MacPherson DW, Gushulak BD, Baine WB, et al. Population mobility, globalization, and antimicrobial drug resistance. Emerg Infect Dis 2009;15:1727-32.

12 Andersen S, Knudsen J. A managed multidisciplinary program on multi-resistant Klebsiella pneumoniae in a Danish University Hospital. BMJ Qual Saf 2013;22:907-15.

13 Wertheim HF, Chandna A, Vu PD, et al. Providing impetus, tools, and guidance to strengthen national capacity for antimicrobial stewardship in viet nam. PLoS Med 2013;10:e1001429.

14 Shekelle PG, Pronovost PJ, Wachter RM, et al. The top patient safety strategies that can be encouraged for adoption now. Ann Intern Med 2013;158(5 Pt 2):365-8.

15 Charani E, Castro-Sanchez E, Sevdalis N, et al. Understanding the Determinants of Antimicrobial Prescribing Within Hospitals: The Role of "Prescribing Etiquette". Clin Infect Dis 2013;57:188-96. 